\title{
Last Upgrades of Roman Pot Electronics
}

\author{
${ }^{1)}$ Richard Linhart, ${ }^{2)}$ Vjačeslav Georgiev \\ Faculty of Electrical Engineering \\ University of West Bohemia \\ Pilsen, Czech Republic \\ ${ }^{1)}$ rlinhart@kae.zcu.cz, ${ }^{2)}$ georg@kae.zcu.cz
}

\author{
Mirko Berretti ${ }^{3)}$, Helsinki Institute of Physics, \\ Helsinki, Finland and CERN, Geneva, Switzerland \\ and Marco Bozzo ${ }^{4)}$, INFN Sezione di Genova, \\ Genova, Italy; \\ 3) mirko.berretti@gmail.com, ${ }^{4)}$ marco.bozzo@cern.ch,
}

\begin{abstract}
This article presents the recent (2017-2018) electronics modifications for the upgrade of the TOTEM roman pot detectors at the CERN LHC. To improve the timing accuracy and reliability the new Roman Pot detector configuration utilizes both DSDD (Double Side Diamond Detector) and silicon (UFSD, Ultra Fast Silicon Detector) mounted on hybrid boards. The electronics described was designed, built and tested by the authors in Faculty of Electrical Engineering in Pilsen, Department of Applied Electronics and Telecommunications, University of West Bohemia and by the TOTEM experiment at CERN. With the new detector setup, timing measurements with resolution down to tens of picoseconds are possible.
\end{abstract}

Keywords-component; semiconductor radiation detectors; particle detectors; diamond detector, ultra fast silicon detector

\section{INTRODUCTION}

The physics program of the TOTEM (TOTal cross section, Elastic scattering and diffraction dissociation Measurement at the LHC) experiment is dedicated to the precise measurement of the proton-proton interaction cross section, as well as to the in-depth study of the proton structure that is still poorly understood at the dynamical level. The study of the physics processes in the region very close to the particles beam (forward region) is complementary to the programs of the LHC general-purpose experiments and requires appropriate detectors. All the detectors of the experimental apparatus will detect charged particles emitted by the proton-proton collisions in the IP5 interaction point and will have trigger capabilities that will allow an online selection of specific events.

\section{THE ROMAN POT DETECTOR}

The Roman Pots (RPS) are special movable vacuum pipe sections supported by bellows to house devices that can thus be positioned very close to the circulating beams; before the measurements the RPS are moved closest to the circulating beams by compressing the bellows. They are called 'Roman' because they were first used by a group of Italian physicists from Rome, in the early 1970 s, to study similar physics at the CERN's ISR (Intersecting Storage Rings). They are called 'Pots' because the delicate detectors, able to localize the trajectory of protons close to the beam within $1 \mathrm{~mm}$ (with a precision of around 20 micrometers), were traditionally housed in cylindrical vessels. In the TOTEM experiment, every Roman Pot station is composed of two units, separated by a distance of $\sim 4$ meters, each unit consisting of two pots in the vertical plane approaching the beam from above and below, and one that moves horizontally. They are placed very far from the interaction point (about 147m and 220m), on both sides of the IP5 interaction point.

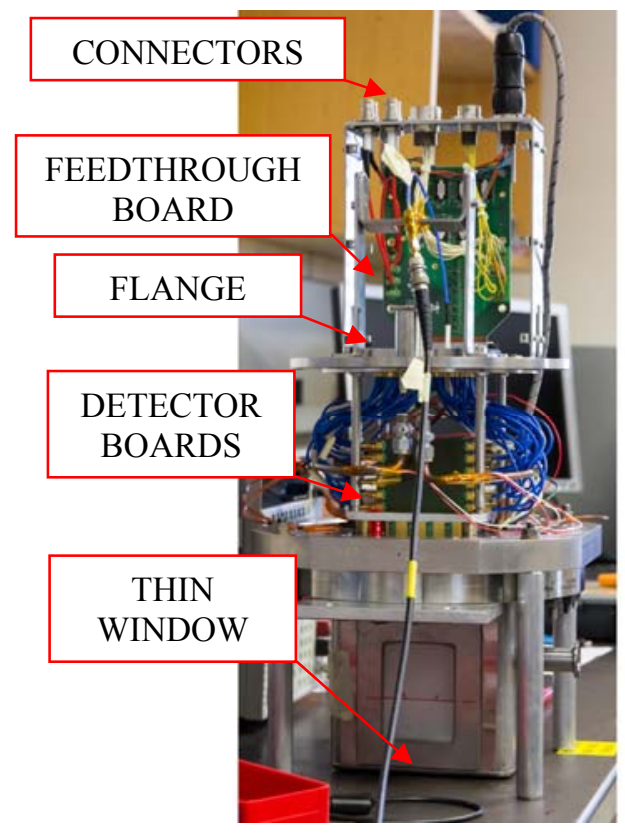

Figure 1. A fully assembled detector on the test stand during laboratory tests. Signals, LV and HV are connected from the top.

Each Roman Pot may house up to four detector boards with both the detector (diamond or UFSD) and the amplifier chain to shape the signals; for this reason they are called hybrid boards. A completed description of the system can be found in [1]. The sensor used to detect the passage of a charged particle is mounted on one edge of the board (the left side if looking at Fig. 4). Similar boards are used for different configurations of particle detectors, in particular single or double side diamonds configuration [2,3] or the Ultra Fast silicon Detector (UFSD) [4]. . The boards provide impedance matching, signal amplification, high voltage filter and temperature sensors. The novelty of the design is very a high gain, very high transition rates, low jitter and stability.

Detector boards are mounted in the main Roman Pot chamber, in a secondary vacuum approximately 50 mbar. The most critical part of the Roman Pot is the thin window, 200 micron thick steel foil between the Roman Pot secondary vacuum, and the primary vacuum of the LHC beam pipe. The detector board frame is cooled down to $-20 \mathrm{C}$ by a heat pump. All electronic parts are in contact with the cooling system to avoid overheating problems. The Roman Pot is kept at 50 mbar and the electric strength of air is then 4 
times lower than at atmospheric pressure. The diamond detector for which the bias voltage can be up to $1 \mathrm{kV}$ required a particular attention to obtain a good high voltage isolation. Requirements were less stringent for the UFSD detectors that need a lower bias voltage of approx. $200 \mathrm{~V}$.

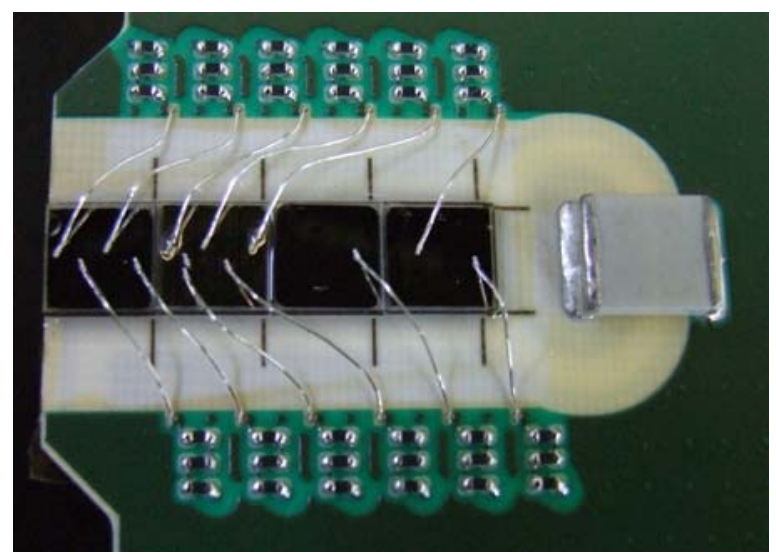

Figure 2. Diamond detector on the horizontal detector board. Four diamond chips, each $4.5 \times 4.5 \mathrm{~mm}$, are divided into 12 pixels. On the left one sees a high voltage capacitor. The visible resistors are for the amplifier feedback network.

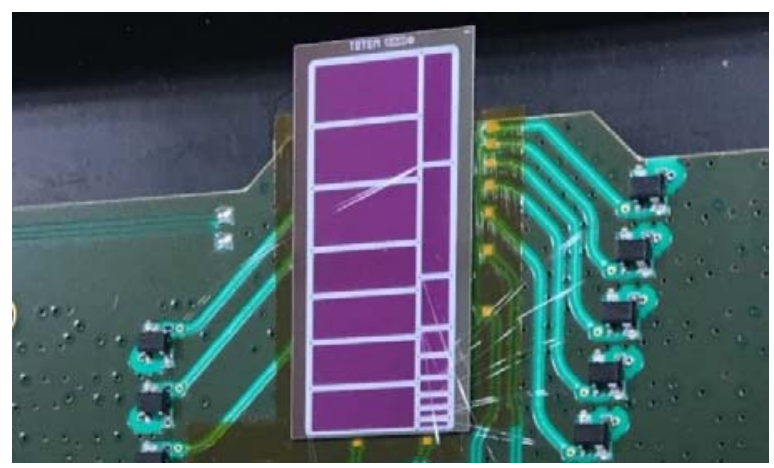

Figure 3. The UFSD detector on an early version of the detector board. The chip is divided and connected into 12 pixels [4].

\section{THE DETECTOR BOARD}

Three main types of detector boards have been developed for use in Roman Pots. Two types are diamond detector boards, (called vertical and horizontal). The last board type is UFSD. All boards contain 12 identical signal amplifier chains for the 12 pixels of the detector. The 48 output signals from the 4 boards inside one RPS are routed through a square vacuum flange with $49(7 \times 7)$ SMP connectors laser welded to the stainless steel plate.

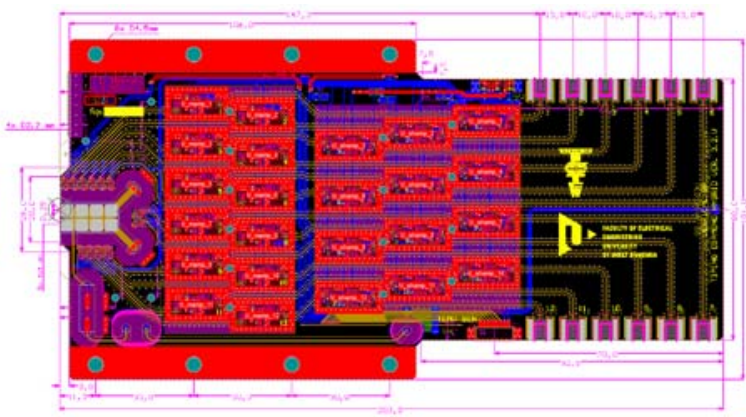

Figure 4. Vertical diamond detector board overview. The detector is on the left, output connectors on the right side.

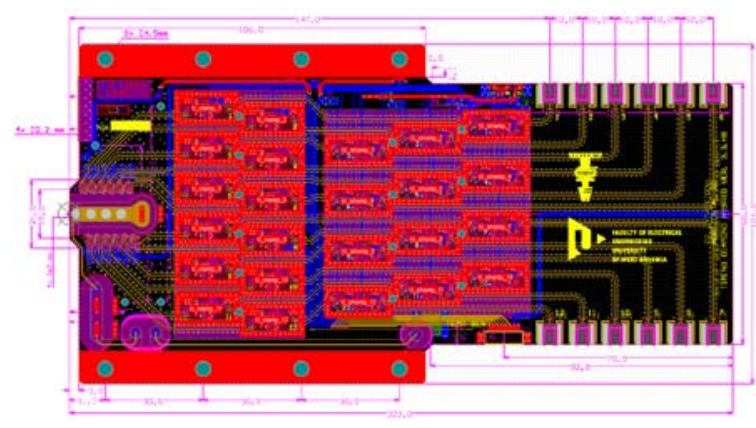

Figure 5. Horizontal diamond detector board overview. The detector is on the left, output connectors on the right side.

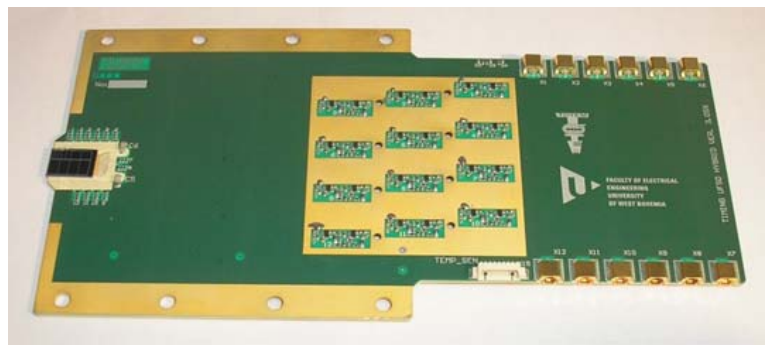

Figure 6. The UFSD detector board. Center block of cells of the flat amplifier is missing on UFSD boards, since the UFSD detector has already a gain of its own [4]

The signal chain on the diamond detector boards consists of 5 stages. The preamplifier, for the impedance matching between the diamond detector (very high impedance) and the $50 \Omega$ transmission lines. Its gain and the frequency response is critical for the noise ratio. After the preamplifier, the signal is fed to the main (flat) amplifier. This is a MMIC amplifier with flat amplitude and phase response on a defined frequency range. The third stage is an attenuator, which improves impedance matching of the next stage and sets the signal amplitude, for which the next stage works best. Last two stages are silicon transistor based amplifiers with impulse response optimized for best signal to noise ratio. The final stage is the transmission line (cable) driver. Because of that, these last stages are often called a shaper and a booster. The stable twelve channels with such a big gain on the place of $11760 \mathrm{~mm}^{2}$ is real state of the art.

All the electronic circuits are concentrated in small cells, which can be easily covered by aluminium shielding boxes. Transmission lines between cells are guided inside the board as $50 \Omega$ strip lines always separated by two rows of ground via holes. Thanks to that, there are no problems with crosstalk or unwanted feedback between circuits inside the board.

TABLE I. DIAMOND DETECTOR BOARD PARAMETERS

\begin{tabular}{|c|l|}
\hline Power supply & $2.0 ; 5.0 ; 3.3 \mathrm{~V}$ \\
\hline Power requirements & $6.2 \mathrm{~W}$ \\
\hline High voltage max. & $1000 \mathrm{~V}$ \\
\hline Output peak voltage & $1 \mathrm{~V}$ \\
\hline Output noise voltage & $12 \mathrm{mV}$ RMS \\
\hline Current gain & $84 \mathrm{~dB} @ 1 \mu \mathrm{A}$ input peak \\
\hline
\end{tabular}

The UFSD detector boards differ from the diamond detector boards. The main difference is missing flat amplifier and modified last two stages. 
Signals from Roman Pots are connected by coaxial cables to the instrumental rack, where they are converted into digital form using NINO-8 [5] chips and measured by High Precision TDC or sampled by the Orsay developed SAMPIC [6] Devices.

TABLE II. UFSD DETECTOR BOARD PARAMETERS

\begin{tabular}{|c|l|}
\hline Power supply & $2.2 ; 5.0 ; 2.4 \mathrm{~V}$ \\
\hline Power requirements & $3.5 \mathrm{~W}$ \\
\hline High voltage max. & $250 \mathrm{~V}$ \\
\hline Output peak voltage & $0.1 \mathrm{~V}$ \\
\hline Output noise voltage & $3 \mathrm{mV} \mathrm{RMS}$ \\
\hline Current gain & $48 \mathrm{~dB} @ 32 \mu \mathrm{A}$ input peak \\
\hline
\end{tabular}

\section{THE FEEDTHROUGH BOARD}

The feedthrough board is a special 4 layer board, which provides all power distribution lines and diagnostic signals inside the Roman Pot main chamber which is kept under vacuum. The board is glued into special flat flange fixed in the Roman Pot cover.

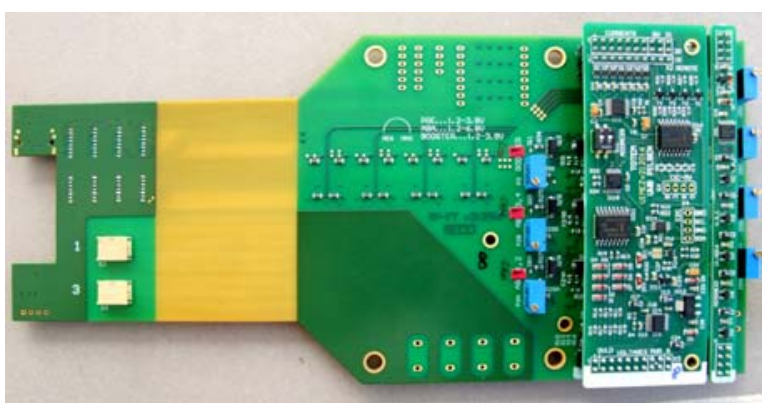

Figure 7. The Feedthrough board with mezzanines inserted in.

The small, inner part of the board contains connectors for the detector boards, plus other system monitoring devices like an air pressure sensor and RADMON, the radiation sensor module. The power distribution consists of 4 high voltage channels (one for each hybrid board), and 7 low voltage rails. High voltage leads are isolated from each other and from the ground and use special high voltage SHV connectors. The low voltage lines are connected by means of 7-pin connectors from Microblade. Other connectors are used for the 4 PT100 temperature sensors mounted on each hybrid. Other 4 wires PT1000 sensors are present on the input and output of the cooling pipe to monitor the proper coolant flow,

The part of the feedthrough board outside the RPS main chamber contains low voltage power regulators and connection fields, which allow to select the temperature sensor of each detector board that will be monitored will be monitored.

The power supply is designed for an input voltage of 6 to $8 \mathrm{~V}$, max $5 \mathrm{~A}$ to be provided by the TOTEM infrastructure. For best reliability for use in high radiation environment highly integrated logic, or FET based components should not be used on the Feedthrough board and all the voltages are regulated by special radiation hardened LDOs type LHC4913. Voltage levels are set manually using precision multi turn trimmers. There are $7 \mathrm{LDO}$ voltage regulators, 4 are for preamplifiers, each detector board have its own low voltage channel. Next 2 LDOs are for $5 \mathrm{~V}$, main (flat) amplifiers. Two detector boards share one LDO, which better fits the current requirements. Last LDO regulator is common for the output stages of all the detector boards.

The feedthrough board also contains power switches based on low saturation voltage BJT transistors, which can be used in conjunction with the LDO control pins to switch a selected detector board off. This feature can be useful in case of bad radiation damage of components on one board that could eventually shorts the power supply. The experiment then can continue with the other detector boards.

\section{REMOte ACCESS MeZzAnines}

Once the detector is installed in the LHC tunnel cannot be accessed for long periods. We have then designed a mezzanine module to the feedthrough board, for added flexibility in setting-up, debugging, remote monitoring and control capabilities. This is also particularly useful in the lab and test particle beams to study and optimize the detector timing response on bias voltages and on temperature. The novelty of design assuring matching of channels is selection of transistors at first stage and separate bias voltage settings for individual stages.

Two special modules (mezzanines) were developed and built. The first one is called U/I mezzanine, because it provides remotely via an I2C link the value of voltages and currents on all low voltage power lines. It also can switch individual detector boards on and off remotely and read overload indicators from all LDOs.

Encouraged by the useful performance of this, we have developed a second mezzanine, the Remote Control mezzanine that works on the same bus address selected for the U/I mezzanine and contains 5 DAC converters, which are used to modify the LDO's output voltage remotely. This proves very useful to speed up the optimization of each hybrid board. If one is afraid of possible radiation damage in the increased radiation now existing in the LHC, after the best voltage settings are found, the remote mezzanine can be removed, the settings applied by adjusting the trimmers and then Feedthrough board can operate as a simple radiation resistant power supply.

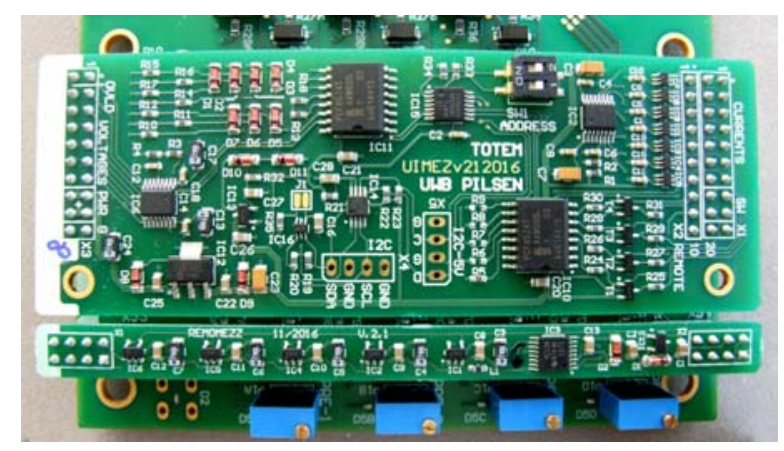

Figure 8. The remote access mezzanines. The U/I monitoring mezzanine (top) and the Remote Control mezzanine (bottom). 


\section{ACCESSORIES}

Development of the many types of boards is performed in different laboratories from the participating universities and to simplify laboratory testing of electronic parts and for test beams, two small additional boards were designed and built. The first one works like a simple power supply with LDO voltage regulators. Output voltage of two branches can be set using precision multi turn trimmers and is protected against overload and short. The simplification comes when one is working on one board and is not in a well-equipped laboratory but in the field: it can be powered from table power supply or also from any common USB mobile phone wall adapter.

The second device is an USB to I2C converter. Its purpose is to setup and diagnose of the Roman Pot electronics in the laboratory or at test beams, where the TOTEM control infrastructure is not yet available. On the USB side, the common chip FT232RL was chosen since it is supported by almost all operating system. For example for Linux, often used in CERN, one doesn't need to install any special driver. On the I2C side, there is a variable level converter, which can be set to any common voltage, $1.8,3.3,5.0 \mathrm{~V}$ or $2.5 \mathrm{~V}$ to emulate the TOTEM I2C interface provided by the CCU25 chips. The pullup resistors or I2C accelerator (active current source) can be switched on the bus. The heart of the device is the microcontroller ATmega328 with the Arduino firmware whose usage is very easy, requiring no special programming experiences to write scripts or to modify existing Arduino scripts to control and read the Roman Pot electronics

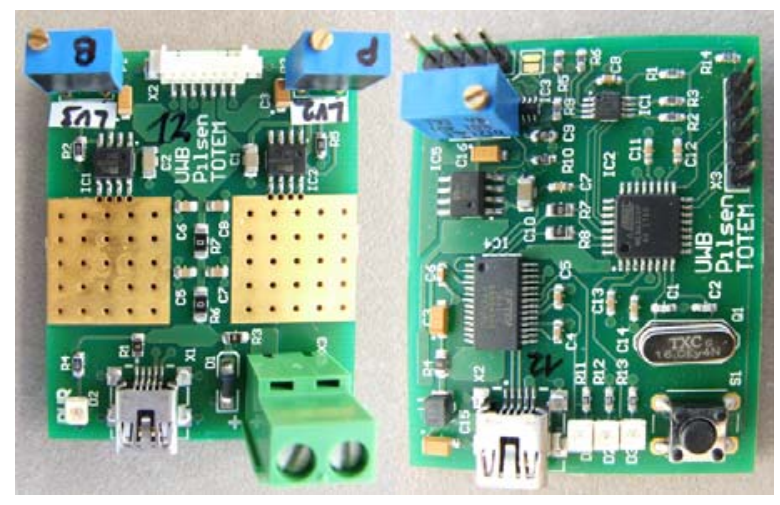

Figure 9. Accessory devices. The single board power supply (left) and TOTEM I2C interface (right).

The Arduino code is simple, accessible and the IDE is available on all common operating systems.

\section{CONCLUSIONS}

All boards were extensively tested and optimized in the $\mathrm{H} 8$ beam tests facility at CERN, before installing them in the RP of the TOTEM experiment in the LHC tunnel in April 2017. Tables of the optimized parameters have been presented above. The timing resolution for the timing telescopes in the LHC for a max luminosity beam has been found to be $\sim 80$ ps for a telescope of 3 diamond planes and one UFSD as expected. The special requirements as low power, high speed and robust design were met at all detector boards.

\section{ACKNOWLEDGMENT}

The work has received financial support from the projects LM2015058, LTT17018, TE01020455

\section{REFERENCES}

[1] M.Berretti (for the TOTEM Collaboration), "The timing upgrade project of the TOTEM Roman Pots detectors", Published in Nucl.Instrum.Meth. A824 (2016).

[2] G. Antchev (TOTEM Collaboration), "Diamond Detectors for the TOTEM Timing Upgrade", published in JINST 12 (2017) no.03, $\mathrm{P} 03007$

[3] M. Berretti et al, "Timing Performance of a Double Layer Diamond Detector", Published in JINST 12 (2017) no.03, P03026

[4] R. Arcidiacono et al, "Test of Ultra Fast Silicon Detectors for the TOTEM upgrade project", Published in JINST 12 (2017) no. $03, \mathrm{P} 03024$

[5] F. Anghinolfi et al, "NINO: An ultra-fast and low-power front-end amplifier/discriminator ASIC designed for the multigap resistive plate chamber", Published in Nucl.Instrum.Meth. A533 (2004) 183-187

[6] E. Delagnes et al, "Reaching a few picosecond timing precision with the 16-channel digitizer and timestamper SAMPIC ASIC", Published in Nucl.Instrum.Meth. A787 (2015) 245-249 